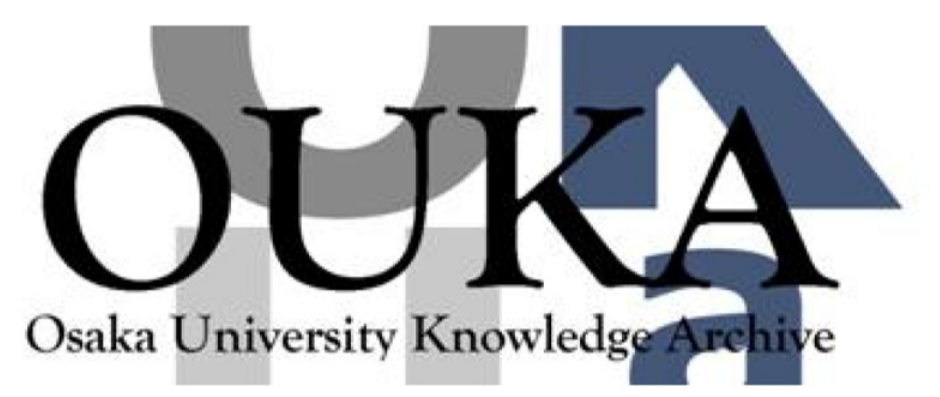

\begin{tabular}{|c|c|}
\hline Title & $\begin{array}{l}\text { Anisotropy and pressure dependence of the upper } \\
\text { critical field of the ferromagnetic } \\
\text { superconductor UGe2 }\end{array}$ \\
\hline Author (s) & Sheikin, I.; Huxley, A.; Braithwaite, D. et al. \\
\hline Citation & Physical Review B. 64(22) p.220503_1-p.220503_4 \\
\hline Issue Date & $2001-11$ \\
\hline oaire:version & VoR \\
\hline URL & https://hdl. handle. net/11094/2920 \\
\hline rights & $\begin{array}{l}\text { Sheikin, I., Huxley, A., Braithwaite, D., } \\
\text { Brison, J. P., Watanabe, S., Miyake, K., } \\
\text { Flouquet, J.', Physical Review B, 64, 22, } \\
\text { 220503, 2001-11. "Copyright } 2001 \text { by the } \\
\text { American Physical Society." }\end{array}$ \\
\hline Note & \\
\hline
\end{tabular}

Osaka University Knowledge Archive : OUKA

https://ir. Library. osaka-u. ac. jp/

Osaka University 


\title{
Anisotropy and pressure dependence of the upper critical field of the ferromagnetic superconductor $\mathrm{UGe}_{2}$
}

\author{
I. Sheikin ${ }^{1, *}$ A. Huxley, ${ }^{1}$ D. Braithwaite, ${ }^{1}$ J. P. Brison, ${ }^{2}$ S. Watanabe,${ }^{3}$ K. Miyake,${ }^{3}$ and J. Flouquet ${ }^{1}$ \\ ${ }^{1}$ Département de Recherche Fondamentale sur la Matière Condensée, SPSMS, CEA/Grenoble, 17 rue des Martyrs, \\ 38054 Grenoble Cedex 9, France \\ ${ }^{2}$ Centre de Recherches sur les très Basses Températures, CNRS, BP166, 38042 Grenoble Cedex 9, France \\ ${ }^{3}$ Department of Physical Science, Graduate School of Engineering Science, Osaka University, Toyonaka, Osaka 560-8531, Japan
}

(Received 6 September 2001; published 12 November 2001)

\begin{abstract}
The temperature and pressure dependence of the upper critical field, $H_{c 2}$, of the ferromagnetic superconductor $\mathrm{UGe}_{2}$ is reported for fields applied along all three crystallographic axes. For fields parallel to the easy magnetic $a$ axis, the relationship between an unusual reentrant behavior of $H_{c 2}$ and a field-induced transition associated with a change in the electronic density of states is reviewed. For transverse field directions a significant evolution in the behavior of $H_{c 2}$ with pressure is found. As the pressure is decreased the dependence of $H_{c 2}$ on temperature for fields along the crystal's $c$ axis acquires a positive curvature that extends from the critical temperature, $T_{c}$, down to almost the lowest temperature measured $\left(T_{c} / 10\right)$ where $H_{c 2}$ exceeds the usual weak coupling paramagnetic and orbital limits.
\end{abstract}

DOI: 10.1103/PhysRevB.64.220503

PACS number(s): 74.70.Tx, 74.25.Dw, 75.50.Cc

$\mathrm{UGe}_{2}$ is a material in which superconductivity and itinerant ferromagnetism have been demonstrated to coexist ${ }^{1,2}$ in single crystals with a Curie temperature that is substantially higher than the superconducting transition temperature $\left(T_{c}\right.$ $\sim 0.8 \mathrm{~K}$ and $T_{\text {Curie }} \sim 35 \mathrm{~K}$ at $12 \mathrm{kbar}$ ). Both states exist over a relatively wide range of applied pressures (11-16 kbar) and apparently disappear simultaneously at an upper critical pressure, $P_{c} \sim 16$ kbar.

Theoretically, the possibility of finding spin triplet superconductivity near to a ferromagnetic quantum critical point was pointed out a long time ago. ${ }^{3,4}$ However, the original scenario considered the divergence of magnetic fluctuations close to a second order quantum critical point, while it has been argued ${ }^{2,5}$ that in $\mathrm{UGe}_{2}$ the ferromagnetic transition becomes first order just below $P_{c}$. Although the associated ferromagnetic fluctuations could still favor the formation of spin triplet Cooper pairs, the maximum interaction obtainable is less than for a second order transition. Experiments, however, suggest that there could be a further phase boundary within the ferromagnetic state., ${ }^{5,6}$ Even though the associated order parameter has not been identified, the observation that the superconducting critical temperature is highest near to the critical pressure $\left(P_{x} \sim 12.5 \mathrm{kbar}\right)$ necessary to suppress the transition suggests that it is intimately related to the pairing interaction. Whatever the pairing mechanism, odd-parity spin triplet superconductivity might be expected since the spin majority and minority Fermi-surfaces are very strongly split in the ferromagnetic state. Since time invariance is already broken by the ferromagnetic order, the superconducting state is necessarily nonunitary, ${ }^{7}$ as suggested from measurements of the flux-flow resistivity. ${ }^{2}$ This situation resembles that of the A1 phase of superfluid ${ }^{3} \mathrm{He}$. Differences from the case of ${ }^{3} \mathrm{He}$ are expected since apart from the obvious change to charged particles, the presence of a strong crystal field can modify the orientation and symmetry of the order parameter.

Measurements of the upper critical field provide a powerful method to help confirm whether the superconductivity is indeed spin triplet. Such measurements have previously led to a hypothesis of triplet pairing in the heavy fermion superconductor $\mathrm{UPt}_{3}$ (Refs. 8-11) and in the quasi-onedimensional organic compound (TMTSF) ${ }_{2} \mathrm{PF}_{6} \cdot{ }^{12,13}$

Our measurements of $\mathrm{H}_{c 2}$ along the three principal crystallographic directions of orthorhombic $\mathrm{UGe}_{2}$ cover three different pressure ranges: just below and above $P_{x}$ and close to $P_{c}$. The measurements were performed in a nonmagnetic piston-cylinder-type pressure cell. $H_{c 2}$ was determined from the electrical resistivity measured by the usual four terminal method with an ac current $(10$ or $100 \mu \mathrm{A}$ at $11.7 \mathrm{~Hz})$ passed along the length of the sample and parallel to its $a$ axis. $H_{c 2}$ was taken as the midpoint of the superconducting transition crossed by either changing the field (several examples are shown in the inset of Fig. 1) or the temperature; our conclusions do not depend on the particular choice of criteria, except for $H \|_{c}$ at $15 \mathrm{kbar}$, where there was a strong dependence on field history, which will be described later. $H_{c 2}$ was measured parallel to the $a$ and $b$ directions at exactly the same pressures without heating the cryostat above liquid helium temperatures. To make measurements with $H \|_{c}$ it was necessary to warm the cryostat and pressure cell to room temperature and change the magnet. This thermal cycle gave rise to a small change of pressure (determined by measuring the superconducting transition of a tin strip in the cell prior to application of any field), that was sufficient to change slightly the superconducting critical temperature of the $\mathrm{UGe}_{2}$ crystal. To facilitate comparison we therefore show in Fig. 1 the temperature dependence of the upper critical field normalized to $T_{c}$ for all three principal orientations of the magnetic field plotted against $T / T_{c}$ for the three distinct pressure ranges considered. The corresponding values of $T_{c}$ are given in the figure caption, while the zero field transitions have been reported elsewhere. ${ }^{14}$

We discuss first $H_{c 2}^{a}$, the upper critical field for a field applied parallel to the easy magnetization direction ( $a$ axis), since a field applied along this direction gives rise to distinct 


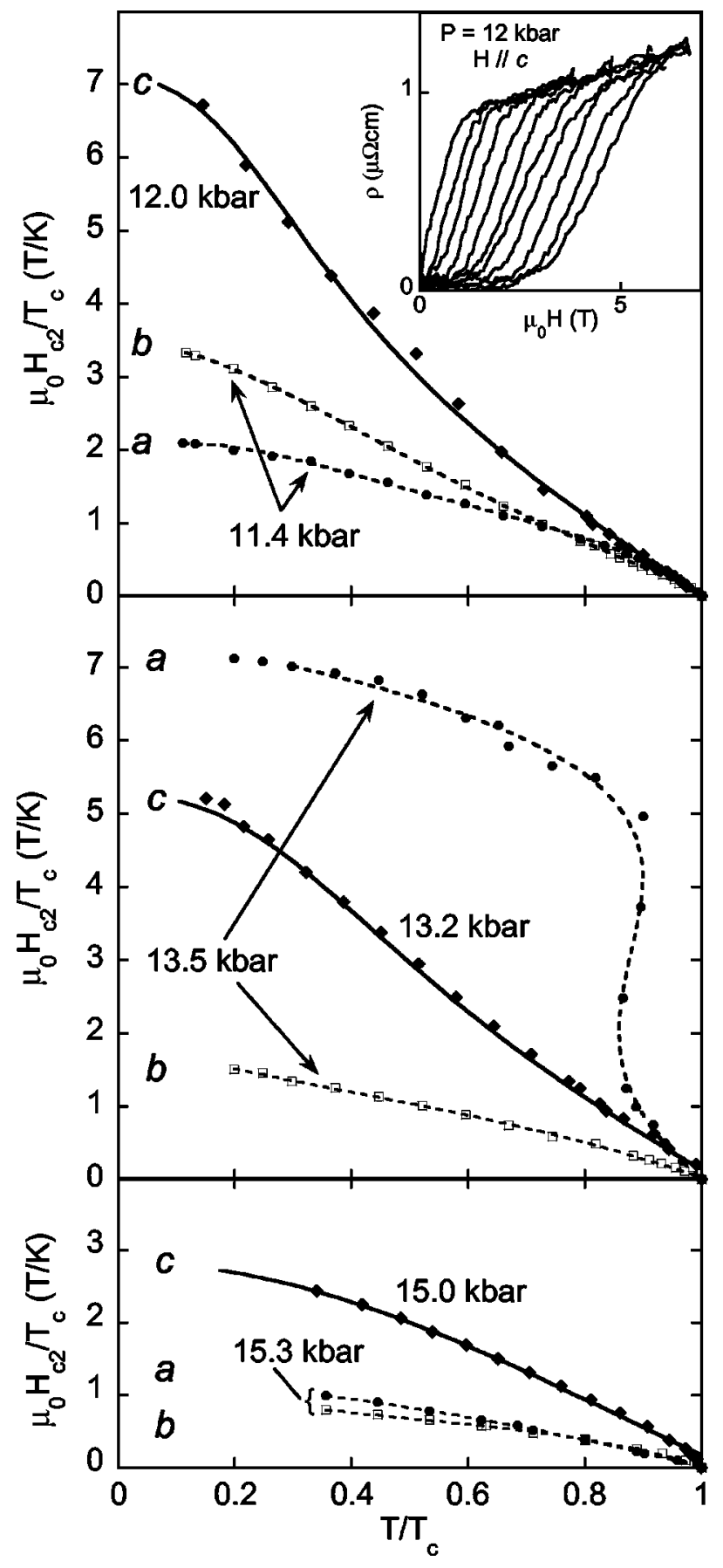

FIG. 1. The upper critical field normalized to the superconducting transition temperature, $H_{c 2} / T_{c}$, is plotted against temperature, $T / T_{c}$, for applied fields parallel to the $a, b$, and $c$ axes. The top and middle panels are, respectively, for pressures slightly below and above $P_{x}$, while the bottom panel corresponds to a pressure close to the critical pressure to suppress ferromagnetism (and superconductivity), $P_{c}$. The critical temperatures at the various pressures are $0.757,0.685,0.466,0.403,0.258$, and $0.225 \mathrm{~K}$ at $11.4,12,13.2$, $13.5,15$, and $15.3 \mathrm{kbar}$. The solid lines are fits to a strong coupling calculation described in the text, while the dashed lines serve only to associate the points. The inset shows resistivity versus magnetic field at temperatures from 0.1 to $0.6 \mathrm{~K}$ in steps of $0.05 \mathrm{~K}$ at $12 \mathrm{kbar}$ for $H \| c$. phenomena in the normal state, and therefore requires special consideration. In zero magnetic field, just below $12.5 \mathrm{kbar}$ there is a sharp transition in the temperature dependence of the normal state resistivity and magnetization at a temperature $T_{x}$ [the latter is also visible in a neutron study at $9.5 \mathrm{kbar}$ where $T_{x}=15 \mathrm{~K}$ (Ref. 2)]. $T_{x}$ decreases with pressure and vanishes at $P_{x}=12.5 \mathrm{kbar}$. There is a drop in the residual resistivity $(20 \%)$, a sharp $(300 \%)$ increase in the coefficient of the temperature dependent part of the resistivity (which remains quadratic at low temperature), ${ }^{2}$ and an increase in the low temperature specific heat ${ }^{15}$ as $P$ is increased through $P_{x}$. These observations suggest that the transition marks a change of phase to a state with a higher electronic density of states above $P_{x}$. Although such observations are consistent with the suppression of a supposed charge- and spin-density wave (CDW/SDW) with pressure and temperature, no direct evidence (e.g., from neutron scattering) has been forthcoming to confirm such a hypothesis. A CDW necessary implies a SDW because of the large splitting between the majority and minority spin Fermi surfaces in the ferromagnetic state. In this case an increase of magnetization $M(T)$ below $T_{x}$ could arise as a result of mode coupling between $M, S_{\vec{Q}}$ and $N_{-\vec{Q}}$, where $S_{\vec{Q}}$ and $N_{-\vec{Q}}$ are the SDW and CDW order parameters with ordering vectors $\pm \vec{Q} \cdot{ }^{16}$

The consequence of the above for $H_{c 2}^{a}$ follows from the sensitivity of $T_{x}$ to a magnetic field parallel to the easy axis. The superconducting transition temperature is determined in a strong coupling formalism by the spectral weight of the Bose excitations that mediate the pairing interaction. In a simplistic model this can be described by a Lorentzian function, $g^{2} F(\omega)=\lambda(\Gamma / \pi)\left[\omega /\left(\omega^{2}+\Gamma^{2}\right)\right]$. Then the number of parameters is reduced to three: a coupling constant, $\lambda$, the Fermi energy (assumed constant), and the Lorentzian width, $\Gamma$, of this distribution. With the approximation that the same Bose spectrum accounts for the entire renormalization of the electronic density of states from its value at $P=0, \lambda(P)$ is simply proportional to the excess specific heat as a function of pressure, which can be estimated from the data of Tateiwa et $a l .{ }^{15} \Gamma(P)$ is then determined by the pressure dependence $T_{c}(P)$. To understand the measurements of $H_{c 2}^{a}(T), P$ is replaced by $\widetilde{P}(H)$ obtained by inverting the relationship $T_{x}(P, H)=T_{x}(\widetilde{P}, 0)$ based on the field dependence of $T_{x}$ at 11 and $13.5 \mathrm{kbar}$ and the zero field dependence of $T_{x}(P){ }^{14}$ The upper critical field at different pressures can then be calculated. The results of such a calculation are shown in Fig. 2. Despite the oversimplistic nature of the above analysis, it is apparent that the strange discontinuous behavior of $H_{c 2}^{a}$ for $P$ just above $P_{x}$ seen experimentally at $13.5 \mathrm{kbar}$ is qualitatively reproduced by this model and can therefore be attributed to a rapid evolution of $\Gamma$ and $\lambda$ as the critical condition $T_{x} \rightarrow 0$ is approached by application of a field. By the same argument there should also be an enhancement of $H_{c 2}^{a}$ at $15.3 \operatorname{kbar}\left(P>P_{x}\right)$ and a small depression of $H_{c 2}^{a}$ at 11.4 kbar $\left(P<P_{x}\right)$ at low temperatures. This is consistent with the data: at $11.4 \mathrm{kbar} H_{c 2}^{a}(T)$ has a stronger curvature than $H_{c 2}^{b}(T)$ at low temperature and $H_{c 2}^{a}(0)<H_{c 2}^{b}(0)$, whereas the situation is reversed at 15.3 kbar. No evolution 


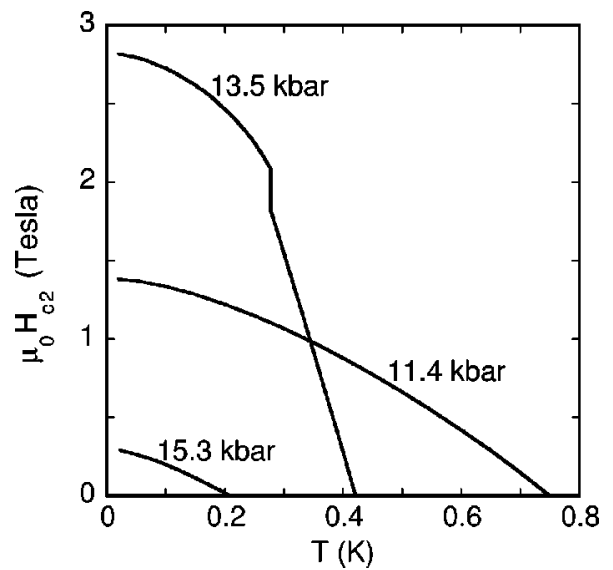

FIG. 2. $H_{c 2}(T)$ for $H \| a$ calculated taking into account the field/ pressure dependence of the transition at $T_{x}$ (see text for explanation).

of $T_{x}$ for applied fields perpendicular to the easy axis has been observed and the above considerations therefore will not modify the form of $\mathrm{H}_{c 2}$ for these directions.

After accounting for the particular effects described in the previous paragraph, the upper critical field $H_{c 2}^{c}$ is seen to be systematically larger than $H_{c 2}^{b}$ and $H_{c 2}^{a}$ (except perhaps very close to $T_{c}$ ). In our data the critical field refers to the applied field which could differ from the total field by up to $\mu_{0} M$ $<0.2 \mathrm{~T}$ where $M$ is the sample magnetization in the ferromagnetic state. The additional field due to the magnetization of the sample can explain an apparent down turn of the $H_{c 2}$ curves for $H<0.1 \mathrm{~T}$ close to $T_{c}$. To avoid this complication all of the following analysis is restricted to data in fields above $0.1 \mathrm{~T}$, where to a first approximation the difference between the applied field and total field can be neglected. The anisotropy of $d H_{c 2} / d T$ close to $T_{c}$ can then be expressed in terms of an anisotropic effective mass tensor $m_{i j}^{-1} \propto\left\langle v_{f i} v_{f j} \Delta^{2}\right\rangle$ such that $\left(d H_{c 2} / d T\right)_{x} /\left(d H_{c 2} / d T\right)_{y}$ $\propto \sqrt{m_{y y}} / \sqrt{m_{x x}}$ (where $x, y$ are principal axis). The mass tensor depends on the Fermi-surface averages (denoted by \langle\rangle ) of different components of the Fermi velocity $\left(v_{f i}\right)$ and the superconducting gap, $\Delta$. The Fermi-velocity anisotropy can eventually be estimated experimentally from the anisotropy of the temperature dependence of the resistivity in the normal state measured with currents along different crystal directions. From our data the limiting value of $d H_{c 2} / d T$ (deduced from the data above $0.1 \mathrm{~T}$ ) decreases strongly with pressure. The corresponding coherence lengths, $\xi$, at 11-12 kbar are all within $25 \%$ of $100 \AA$. While the anisotropy between the $a$ and $b$ axis is hardly changed at $15 \mathrm{kbar}$, a marked anisotropy with respect to the $c$ axis becomes apparent with $\xi_{a} \approx 210 \AA, \xi_{b} \approx 140 \AA$, and $\xi_{c} \approx 700 \AA$. In addition at $15 \mathrm{kbar}$ there is a large hysteresis in $H_{c 2}^{c}$ on cycling the field. Figure 3 shows that the transition to the normal state in increasing field occurs at a lower field than in a decreasing field. This suggests that there are differently oriented magnetic domains with different apparent $H_{c 2}$ 's depending on the relative alignment of the applied field to the domain direction. In the low pressure magnetic structure the ordered moments are, however, locked to the $a$ axis, whereas the

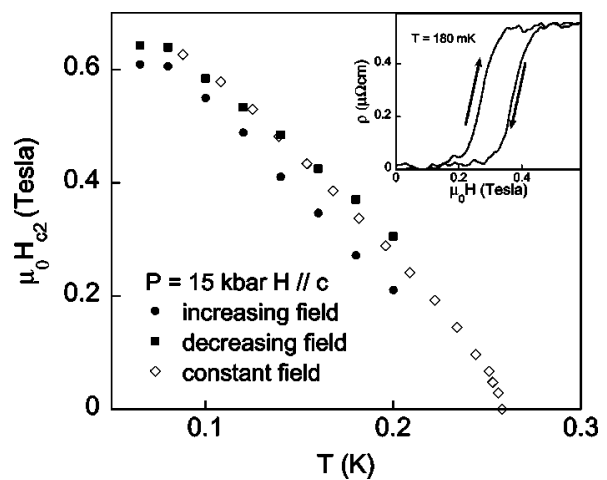

FIG. 3. $H_{c 2}(T)$ for $H \| c$ at 15 kbar determined from both temperature and field sweeps is shown to illustrate the unusual hysteresis (see the inset) between the increasing and decreasing field measurements.

hysteresis for $P \approx P_{c}$ occurs only for $H \| c$. This hints that close to $P_{c}$, a rotation of the moment in the $a c$ plane might occur. The unusual hysteresis is not visible for the other field directions or at lower pressure.

The curves for $H_{c 2}^{c}(T)$ are also remarkable at lower pressures, where they show an unusual positive curvature and $H_{c 2}^{c}(0)$ is much larger than given by both the usual weak coupling orbital and paramagnetic limits. A positive curvature of $H_{c 2}$ has been seen in other superconductors, notably in $\mathrm{Tl}_{2} \mathrm{Ba}_{2} \mathrm{CuO}_{6}{ }^{17}$ the organic material (TMTSF) ${ }_{2} \mathrm{PF}_{6},{ }^{12}$ and in the heavy fermion material $\mathrm{UBe}_{13},{ }^{18}$ although for $\mathrm{Tl}_{2} \mathrm{Ba}_{2} \mathrm{CuO}_{6} H_{c 2}$ does not exceed the paramagnetic limit. (TMTSF) ${ }_{2} \mathrm{PF}_{6}$ is extremely anisotropic and the low dimensionality of the Fermi surface in high fields is proposed to lead to the reestablishment of superconductivity above the usual orbital limit, ${ }^{13}$ although the exact form of the $H_{c 2}$ curve is predicted to be very sensitive to the purity of the material. ${ }^{19}$ The anisotropy of $H_{c 2}$ manifest in this and other organic materials where such explanations might be appropriate is however orders of magnitude larger than for $\mathrm{UGe}_{2}$. Therefore, the explanation put forward for a strong curvature of $H_{c 2}$ in the cubic material $\mathrm{UBe}_{13}$ appears more relevant to the present case. Thomas et al. ${ }^{18}$ proposed that in $\mathrm{UBe}_{13}$ the coupling parameter, $\lambda$, is extremely large which can give both a large $H_{c 2}(0)$ and a positively curved temperature dependence. $H_{c 2}$ is required (as observed in $\mathrm{UBe}_{13}$ ) to saturate and have negative curvature in this model at very low temperatures in contrast to the models that apply to (TMTSF) ${ }_{2} \mathrm{PF}_{6}$. The details of the form of $H_{c 2}$ calculated for strong coupling depend upon the details of the Bose spectrum and its coupling to the electrons to give pairing. However, a simple estimate in an extreme case where the spectrum is a $\delta$ function at finite frequency (an alternative form to the Lorentzian centered at zero frequency considered earlier) can be made as outlined by Bulaevskii ${ }^{20}$ in terms of the coupling parameter $\lambda$, where $\lambda \ll 1$ again gives the weak coupling BCS result. ${ }^{21}$ In such a model with isotropic pairing the form of the curve is completely determined by the low temperature limiting field and the slope of the upper critical field near to $T_{c}$. The calculation should be regarded as illustrative, serving only to give a taste of the forms that might be 
compatible with a more realistic pairing spectrum and taking into account the gap anisotropy. With Bulaevskii's model ${ }^{20}$ we cannot explain the anisotropy of $H_{c 2}$ along different directions (different $\lambda$ 's would be required) but we can get an idea of how the coupling strength might evolve with pressure. Fits to the experimental data for $H_{c 2}^{c}$ are shown in Fig. 1 as solid lines, where the values of the parameter $\lambda$ are 14, 7, and 1.7 at 12, 13.2, and $15 \mathrm{kbar}$, respectively. Unlike for $\mathrm{UBe}_{13}$, good fits are obtained only if Pauli limiting is excluded in the calculation as would be appropriate for certain spin triplet states. For a spin triplet state another higher limiting field occurs due to the effect of the field on the nonzero orbital momentum of the Cooper pairs, ${ }^{22} H_{l}=\left(m^{*} / m\right) H_{p}$ (where $m^{*} / m$ is the effective electron mass compared to the bare mass and $H_{p}$ is the usual paramagnetic limiting field). Since $m^{*} / m$ is at least 3 in the pressure range where superconductivity is observed, this limit is not quite attained.

In this paper we have reported that at pressures close to $P_{x}, H_{c 2}$ significantly exceeds both paramagnetic and orbital limits that would apply to a weakly coupled $s$-wave superconductor. Further, the paramagnetic limit appears to be exceeded even for the case of strong coupling. The lack of paramagnetic limitation argues against a hypothesis of $s$-wave pairing, but is consistent with triplet superconductiv- ity. Although the necessary parameters to make complete calculations including the anisotropy of the pairing interaction and Fermi surface are not yet established, it is clear that the positive curvature of $H_{c 2}(T)$ contrasts with the usual dependence for phonon mediated superconductors, but bears some similarity with the heavy fermion material $\mathrm{UBe}_{13}$, suggestive of a very strong coupling. Thus although phonons could still contribute to pairing, other pairing interactions related to the strongly correlated electronic state might play a significant role. The strength of the coupling appears to decrease rapidly and the coherence lengths become larger as one moves away from the critical pressure $P_{x}$. This relationship is most dramatically demonstrated by the results for $H_{c 2}^{a}$ where the extreme sensitivity of $T_{x}$ to field parallel to the easy axis gives rise to an unusual reentrant behavior of the superconductivity. Finally, close to $P_{c}$ an unusual hysteresis of $H_{c 2}^{c}$ occurs, which suggests that the applied field required to suppress superconductivity is different in differently oriented magnetic domains. The observation of hysteresis only for fields parallel to the $c$ axis, however, is surprising given the Ising-like anisotropy of the magnetism at lower pressures where the ordered moments have been shown to be aligned to the $a$ axis.
*Present address: University of Geneva, DPMC, 24 quai ErnestAnsermet, 1211 Geneva 4, Switzerland.

${ }^{1}$ S. S. Saxena, P. Agarwal, K. Ahilan, F. M. Grosche, R. K. W. Hasselwimmer, M. J. Steiner, E. Pugh, I. R. Walker, S. R. Julian, P. Monthoux, G. G. Lonzarich, A. Huxley, I. Sheikin, D. Braithwaite, and J. Flouquet, Nature (London) 406, 587 (2000).

${ }^{2}$ A. Huxley, I. Sheikin, E. Ressouche, N. Kernavanois, D. Braithwaite, R. Calemczuk, and J. Flouquet, Phys. Rev. B 63, 144519 (2001).

${ }^{3}$ D. Fay and J. Appel, Phys. Rev. B 22, 3173 (1980).

${ }^{4}$ G. G. Lonzarich, in Electron: A Centenary Volume, edited by M. Springford (CUP, Cambridge, 1999), p. 109.

${ }^{5}$ A. Huxley, I. Sheikin, and D. Braithwaite, Physica B 284-288, 1277 (2000).

${ }^{6}$ G. Oomi, K. Nishimura, Y. Onuki, and S. W. Yun, Physica B 186-188, 758 (1993).

${ }^{7}$ K. Machida and T. Ohmi, Phys. Rev. Lett. 86, 850 (2001).

${ }^{8}$ B. Shivaram, T. Rosenbaum, and D. Hinks, Phys. Rev. Lett. 57, 1259 (1986).

${ }^{9}$ H. Suderow, J. P. Brison, A. Huxley, and J. Flouquet, Phys. Rev. Lett. 80, 165 (1998).

${ }^{10}$ C. H. Choi and J. A. Sauls, Phys. Rev. Lett. 66, 484 (1991).

${ }^{11}$ H. Tou, Y. Kitaoka, K. Asayama, N. Kimura, Y. Onuki, E. Yamamoto, and K. Maezawa, Phys. Rev. Lett. 77, 1374 (1996).

${ }^{12}$ I. J. Lee, M. J. Naughton, G. M. Danner, and P. M. Chaikin, Phys. Rev. Lett. 78, 3555 (1997); I. J. Lee, P. M. Chaikin, and M. J. Naughton, Phys. Rev. B 62, R14 669 (2000).
${ }^{13}$ A. G. Lebed, K. Machida, and M. Ozaki, Phys. Rev. B 62, R795 (2000).

${ }^{14}$ J. Flouquet, A. Huxley, I. Sheikin, N. Kernavanois, D. Braithwaite, E. Ressouche, S. Watanabe, and K. Miyake, J. Phys. Soc. Jpn., Suppl. A 70, 14 (2001); A. Demuer, I. Sheikin, D. Braithwaite, B. Fak, A. Huxley, S. Raymond, and J. Flouquet, J. Magn. Magn. Mater. 226-230, 17 (2001).

${ }^{15}$ N. Tateiwa, T. C. Kobayashi, K. Hanazono, K. Amaya, Y. Haga, R. Settai, and Y. Onuki, J. Phys.: Condens. Matter 13, L17 (2001).

${ }^{16} \mathrm{~S}$. Watanabe and K. Miyake, Proceedings of the Conference on Strongly Correlated Electron Systems 2001, Ann Arbor, MI, August 6-10, 2001 [Physica B (to be published)].

${ }^{17}$ A. P. Mackenzie, S. R. Julian, G. G. Lonzarich, A. Carrington, S. D. Hughes, R. S. Liu, and D. C. Sinclair, Phys. Rev. Lett. 71, 1238 (1993).

${ }^{18}$ F. Thomas, B. Wand, T. Luhmann, P. Gegenwart, G. R. Stewart, F. Steglich, J. P. Brison, A. Buzdin, L. Glemot, and J. Flouquet, J. Low Temp. Phys. 102, 117 (1996).

${ }^{19}$ V. P. Mineev, J. Phys. Soc. Jpn. 69, 3371 (2000).

${ }^{20}$ L. N. Bulaevskii, O. V. Dolgov, and M. O. Ptitsyn, Phys. Rev. B 38, 11290 (1988).

${ }^{21}$ T. P. Orlando, E. J. McNiff, Jr., S. Foner, and M. R. Beasley, Phys. Rev. B 19, 4545 (1979).

${ }^{22}$ I. A. Luk'yanchuk and V. P Mineev, Pis'ma Zh. Eksp. Teor. Fiz. 44, 183 (1986) [JETP Lett. 44, 233 (1986)]. 